\title{
EL MOVIMIENTO DE LA ESCUELA MODERNA DE PORTUGAL EN EL AULA DE HOY. UN CASO PRÁCTICO
}

\section{The movement of the Portuguese Modern School in today classroom. A case study}

\author{
Ana Yara Postigo Fuentes \\ Universidad de Málaga \\ Correo-e: anayaratranslator@gmail.com \\ Pedro Francisco González RoA \\ Universidad dos Açores \\ Correo-e: pedrogonzalez@uac.pt
}

Recibido: 28 de marzo de 2019

Envío a informantes: 4 de mayo de 2019

Aceptación definitiva: 23 de junio de 2019

Resumen: En este artículo se presenta una práctica en la actualidad en el aula del Movimiento de la Escuela Moderna en Portugal. En el día a día se ve reflejada la filosofía detrás del Movimiento y su cultura pedagógica en un contexto particular de aprendizaje en el que la docente titular organiza su trabajo cotidiano siguiendo las líneas de esta propuesta pedagógica. Se ha llevado a cabo una observación etnográfica, entrevista a la docente titular y a una docente en prácticas, además de conversaciones informales con ellas, y análisis de documentos de trabajo en el aula para una mayor comprensión de la práctica. Como principales resultados se presentan la actualidad de los principios del Movimiento hoy en día y su planteamiento relevante en el aula.

Palabras Clave: mem; democracia; práctica educativa; participación; autonomía; organización escolar.

Aвstract: On this article we expose a current practice according to the Movement of the Portuguese Modern School. The philosophy behind the Movement and its education culture can be lived in the daily routine of this particular learning context, where 
the teacher organizes her work according to it. It has been carried out an ethnographic observation, an interview with the main teacher and a teacher in practice, on top of informal conversations and an analysis of the work documents in the classroom. As main results, the principles of the Movement are presented as still current and why it is relevant in the classroom.

KEY WORDS: MEM; democracy; educational practice; participation; autonomy; school organization.

\section{Introducción}

\lceil

a Escuela Moderna es un movimiento que se funde con la historia de la educación en Portugal en los últimos cincuenta años (González, 200o) y que sigue teniendo vigencia en la realidad al tratarse de un modelo pedagógico y no un «recetario» o conjunto de metodologías que se pueda aplicar de manera independiente sin una reflexión al respecto.

Una de las características del Movimiento es tratar al profesional de la educación como atento, en un sentido crítico, al contexto educativo y social. Este profesional reconoce el apoyo en el grupo para crecer profesionalmente. Es lo que llamaríamos la autoformación cooperada, en la que se incita a compartir de manera permanente y reflexionar cooperativamente sobre sus prácticas pedagógicas. Estas reflexiones inciden en los materiales que construyen, las técnicas e instrumentos de organización que utilizan y las reflexiones a partir del diálogo permanente con el contexto sociocultural y la realidad del alumnado. De esta manera, el centro de la formación del Movimiento sería la construcción de los saberes profesionales en interacción con sus iguales (González, 2000).

Otro de los aspectos fundamentales es el conocimiento del contexto en el que se desarrolla el Movimiento y la valoración de la intervención en este. Así, los contenidos se estructuran en proyectos que se negocian entre el docente y el alumnado y tienen como punto de partida los saberes extraescolares que provienen de la vida de estos y de sus comunidades. El alumnado se motiva más y aprende mejor cuando viven e indagan problemas reales (Pérez, Soto, Sola y Serván, 2009). La consecuencia es una participación activa en el proceso de planificación, ejecución y regulación del trabajo escolar en un proceso de diálogo negociado activo (Pires, 1998). Este es un ejemplo de ejercicio de democracia directa, que es el principal eje del modelo de educación escolar en la Escuela Moderna Portuguesa (Niza, 1996) y que se construye en el día a día a través de la vivencia en la escuela. Como veremos, es preciso que se construyan modos de organización del trabajo en el aula para poder garantizar una calidad pedagógica que posibilite al alumnado progresar en su itinerario de aprendizaje y construyan los saberes para hacerlos suyos, en base a los valores propios de la democracia, como son la libertad y la igualdad, de los que derivan la tolerancia y el respeto a los demás, por un lado, y la solidaridad y generosidad, por otro (López Melero, 2006). Sabemos que el aprendizaje será más efectivo cuando se respeta la singularidad de cada aprendiz. Es decir, cuando se atienden las diferencias en el desarrollo físico, intelectual, emocional, lingüístico y social de los aprendices, esto es, respetar y atender la diversidad (Pérez et al., 2009). 
Sérgio Niza (Niza, 1992: 47), fundador del MEM, expone los principios estratégicos de estos docentes en su compromiso e intervención social de la educación:

I. Os meios pedagógicos veiculam (em si) os fins democráticos da educação.

2. A atividade escolar, enquanto contrato social educativo, explicitar-se-á através da negociação progressiva dos processos de trabalho que fazem evoluir a experiência pessoal para o conhecimento dos métodos e dos conteúdos científicos e artísticos.

3. A prática democrática de planeamento (atividades e projectos), organização e avaliação e regulação social da vida escolar, partilhada por todos, institui-se em conselho de cooperação.

4. Os processos de trabalho escolar reproduzem os processos sociais autênticos da construção da cultura nas Ciências, nas Artes e no Quotidiano (Homologia de processos).

5. A informação partilha-se através de circuitos sistemáticos de comunicação dos saberes e das produções (ciclos de produção e consumo).

6. As práticas escolares darão sentido social imediato às aprendizagens dos alunos através da partilha dos saberes e das formas de intervenção social.

7. Os alunos intervêm ou interpelam o meio social e integram na aula «Actores» comunitários como fonte de saberes dos seus projetos.

El hecho de que el Movimiento siga vigente se debe a las dimensiones del concepto de cultura en el que se basa el MEM y que expone González (2000):

- En primer lugar, encontramos la berencia pedagógica, es decir, el reconocimiento de la construcción durante décadas de un esforzado trabajo cooperativo que ha servido para encuadrar y sustentar el trabajo pedagógico de los profesionales que forman parte del MEM. Esta cultura, sin embargo, está elaborándose permanentemente, por lo que demuestra flexibilidad en su estructura interna y apertura al contexto en el que se integra.

- Esto nos lleva a la segunda dimensión, que tiene que ver con el ámbito relacional, y que consiste en ese encuentro y diálogo dentro de la comunidad que interviene en la reconstrucción de la cultura y que da un sentido de pertenencia muy fuerte.

- La tercera dimensión tiene que ver con el dinamismo, que se traduce en una disposición para la innovación y disponibilidad para la confrontación honesta que empuja también a esa constante construcción y reconstrucción de la pedagogía.

Con esta investigación nos hemos querido acercar a una experiencia en escuelas donde este modelo está presente en el aula. En el día a día se ve reflejada la filosofía detrás del Movimiento y su cultura pedagógica en un contexto particular de aprendizaje en el que la docente titular organiza su trabajo cotidiano siguiendo las líneas de esta propuesta pedagógica.

\section{Proceso metodológico para recoger y analizar los datos}

La escuela donde se ha realizado la investigación es una institución de primaria y preescolar de 155 alumnos/as, nos concentraremos en el preescolar, nivel educativo en 
el que se realiza la observación. Existen dos clases del mismo nivel que funcionan de manera independiente con diferentes criterios pedagógicos. No existe ningún conflicto en este aspecto, puesto que se entiende que cada docente puede trabajar de manera independiente. En ambas clases los/as alumnos/as tienen una edad comprendida entre tres y seis años. Esto podría sorprender a otras escuelas que estructuran por edades también la etapa preescolar. Sin embargo, es un sistema que funciona en este contexto, puesto que los más mayores ayudan a los más pequeños a adquirir conocimientos, y esta atmósfera hace que crezcan en diferentes valores que permitan la convivencia. Es decir, aproximarse, lo más posible, a un contexto social real. Esto forma parte del compromiso del discurso de la cultura de la democracia y la lucha contra las desigualdades y la segregación (López Melero, 2006). En el aula hay una docente titular que es quien idea y lleva a cabo este proyecto y dos docentes en prácticas que se encuentran en el aula en todo momento y se turnan para liderar las clases de manera semanal.

Se han realizado observación etnográfica sin interacción con el entorno (Goezt y Lecompte, 1988); entrevistas a la docente titular y a una docente en prácticas, además de conversaciones informales con ellas, y análisis de documentos de trabajo en el aula para una mayor comprensión de la práctica. Las numerosas fuentes de información desde diferentes ángulos nos permiten cumplir con el requisito metodológico de la triangulación de datos (Gibbs, 20I2; Simons, 20II).

En este trabajo dedicamos menos espacio a la dimensión de formación cooperada. Los datos recogidos tienen que ver con la bibliografía y las declaraciones de los informantes. Las observaciones seguidas de diálogos con los intervinientes en contextos escolares produjeron bastante material convertido en escrito que es lo que soportó la mayor parte del trabajo aquí presentado.

\section{Tratamiento y análisis de los datos}

El aula está dividida en nueve zonas bien estructuradas y pensadas: ciencias, juegos de alfombra, matemáticas, ordenador, lenguaje escrito, dibujo, biblioteca, casa de muñecas y pintura/modelaje. Los materiales que se encuentran en estas zonas los han aportado la docente titular o los padres, ya que «las partidas económicas para materiales por parte del colegio son pequeñas», según aclara una de las docentes. El horario se estructura en periodos de hora y media entre los que hay una primera pausa de media hora, una hora de almuerzo y después no hay pausa hasta el final de la jornada, que es a las $15: 00 \mathrm{~h}$.

Aunque el horario venga dado por el colegio, las prácticas educativas que suceden en el aula no son azarosas, sino que son pensadas para dar sentido ético al trabajo educativo y se orientan por algunas constantes democráticas del MEM, como son la autoformación cooperada de sus miembros, que podemos observar a través de las reuniones de reflexión que tienen los docentes tras la jornada (docente-docente), en la forma de evaluación que veremos más adelante (docente-alumnado) o en la forma de trabajar del alumnado entre ellos; y la construcción de prácticas democráticas derivadas de las actividades de autoformación. Enseñar requiere colaboración, ayudar a otros y entender qué no saben otros (Tomasello, 2009). Esta capacidad mentalista parece ser uno de los rasgos distintivos en nuestra especie (Premarck y Premarck, 1996). 
Por tanto, estos espacios y las actividades propuestas en ellos han sido cuidadosamente pensados con estas orientaciones y con la mirada puesta en que la ética no puede ser una clase en la que «enseñar valores» como la libertad, la tolerancia, la justicia o la solidaridad. Los valores no se enseñan, han de vivirse, de practicarse. Se tiene entonces claro que la escuela pública se responsabilice de ponerlos en práctica y se traigan al aula para vivirla (López Melero, 2006). Será fundamental comprender en profundidad la naturaleza del aprendizaje para una buena planificación y estructura de los escenarios y actividades y la mejora de la participación del alumnado en la tarea de aprender con un mayor control y responsabilidad sobre el proceso y planificación de su propio aprendizaje (Pérez, Soto, Sola y Serván, 2009). Esta planificación será clave para uno de los pilares claves en el aprendizaje relevante, que es la experimentación del cambio, en el que se expone a los aprendices a diferentes situaciones de aprendizaje o a situaciones cambiantes de un mismo fenómeno. En este proceso, no podemos olvidar que los cambios requieren ser experimentados como tales por los aprendices. Para ello, será fundamental la implicación real de los estudiantes en los contextos, situaciones y experiencias de aprendizaje (Pérez et al., 2009).

A primera hora de la mañana los niños y niñas se quitan los zapatos y se sientan en una rueda en la que pueden compartir lo que quieran si se apuntan en una lista. En el caso de que aún no sepan escribir, se anima a que lo intenten y una de las docentes escribe el nombre de manera correcta al lado, para que poco a poco vayan interiorizando la forma de las letras. Esto es porque para que el alumnado aprenda a leer y, posteriormente, a escribir, tiene que entender la relación de los símbolos gráficos con los sonidos, es decir, cómo se corresponden los grafemas y los fonemas, para un primer momento de decodificación, que sería el proceso básico de asignación de sonido a estímulo visual, y un segundo momento de identificación de las palabras escritas, que implica acceder al significado (Defior, 20I4). Esta actividad intenta incluir acciones desde diferentes vertientes que Clemente y Rodríguez (2014) definirían como buenas prácticas, ya que una característica de los buenos docentes es conjugar elementos desde diferentes enfoques (Pressley et al., 20or). Estas vertientes serían cuatro. En primer lugar, acciones que despierten el interés del alumnado de ser lector, porque le permitirá disfrutar de otras realidades y comunicarse de otra manera con los demás. En este caso, con el resto de sus compañeros/as que ya sí saben escribir. Esto lleva a la segunda vertiente, que son acciones que lleven a la comprensión de que con la escritura se puede representar la realidad como ocurre con el gesto, el dibujo o el lenguaje oral. Al mostrar al alumnado cómo se escribe un conjunto de sonidos que ya conoce, estaremos llevando a cabo las acciones que facilitan adquirir habilidades lingüísticas y metalingüísticas. Posteriormente, todo este conocimiento llevará a un cuarto tipo de acción que encamine a comprender textos.

En el momento de intervención, el resto de los/as compañeros/as escuchan lo que tenga que decir y hacen preguntas al respecto. Después la profesora escribe el plan del día, que al final de la mañana comprobarán si se ha llevado a cabo. Es entonces cuando se dividen en grupos dependiendo de la tarea que vayan a realizar. El encargado de la presencia marca la presencia y entre todos escriben el registro del tiempo. Ambos documentos se usan para trabajar gráficos en el área de matemáticas. La asignación de estas responsabilidades la hace la docente de acuerdo con la necesidad que haya presentado el alumnado con anterioridad. Esta organización, como indicábamos antes, permite garantizar la calidad pedagógica y alterna 
diferentes momentos de trabajo: colectivo del grupo y autónomo, ya sea individual o en pequeño grupo (Pires, 1995).

Además, hoy sabemos que el establecimiento de rutinas y automatismos no necesita la conciencia para funcionar y ser eficaces, por lo que la mayoría de los mecanismos y conexiones cerebrales que vehiculan procesos mentales funcionan por debajo de la conciencia, tanto en la percepción como en la toma de decisiones y en la actuación (Pérez Gómez, 20I2). Estas rutinas que se establecen en el aula cobran especial importancia por lo que Kegan (2010) definió como compromisos ocultos, que son aquellas redes de hábitos y dependencias que no se hacen conscientes, pero que condicionan la vida de las personas o incluso generan comportamientos que impiden alcanzar objetivos de sus vidas.

Estas formas alternas de trabajo (colectivo del grupo y autónomo, ya sea individual o en pequeño grupo) se dan porque, sea cual sea el ámbito del saber en el que nos movemos, para formar este conocimiento reflexivo y consciente se requiere, por un lado, que exista una confrontación, que podríamos definir como compleja y creadora, entre los significados y conocimientos adquiridos previamente de forma poco consciente, y los nuevos significados y conocimientos, que se contrastan mediante la experiencia y la crítica pública. Por otro lado, tanto en el aprendizaje individual como en la investigación en grupo, el suceso más importante es cuando nos abrimos a nuevas formas de ver e interpretar la realidad, discernimos aspectos críticos de los fenómenos y situaciones no considerados hasta el presente y los utilizamos como plataformas de interpretación y actuación innovadoras (Pérez et al., 2009). La distancia crítica requiere, por tanto, el contraste, la pluralidad de alternativas, el cambio de mirada y de posición. Este aspecto está íntimamente relacionado con otra estrategia clave para el desarrollo de aprendizaje relevante, que sería el contraste de perspectivas e interpretaciones de distintas personas y grupos humanos, a través de los que se aprende a identificar y discernir aspectos críticos bien distintos en consonancia con sus contextos y patrones culturales. Esto lleva a una comprensión tanto de las dificultades que otros encuentran como de las estrategias que utilizan para resolver los problemas y dificultades de la realidad (Pérez et al., 2009).

Cuando así corresponda, el alumnado puede decidir a qué va a dedicar su tiempo autónomo. Para gestionarlo, el aula cuenta con un cuadrante en el que figura el nombre de cada niño/a y los diferentes espacios del aula. Cada zona del aula tiene tres oportunidades de ser usada. Cuando un niño/a usa esta zona, debe tacharla. El cuadrante cambia una vez al mes. Este sistema de gestión ofrece la oportunidad de experimentar las diferentes zonas, no solo aquellas que sean más del disfrute del alumno/a. Además, dentro de cada zona, el alumnado continúa su aprendizaje propio de los contenidos, por lo que su aprendizaje es individual y mayoritariamente autónomo, aunque puedan recibir la orientación de las docentes, aprovechando este momento para prestar una ayuda más individualizada y directa a quien lo necesite o para promover la interacción cooperativa entre iguales (González, 2000). Es propio del MEM defender una filosofía que facilite la autoformación y la cooperación entre iguales.

Esta forma de aprendizaje en relación con el entorno tiene que ver con el concepto de enactivismo, concepto acuñado por Francisco Varela, Evan Thompson y Eleanor Rosch (199I), con la idea de resaltar la naturaleza constructivista del aprendizaje del ser humano ligado a su acción. El enactivisimo, por tanto, sería el resultado de unir el constructivismo con la cognición corpórea (Pérez Gómez, 20I2), ya que destaca la 
intervención conjunta e inseparable del cuerpo y la mente. Con este concepto aportan una serie de ideas relevantes para el asunto que tratamos en esta investigación. En primer lugar, el enactivismo recoge la idea de conocer a través de la acción, que incluye la interacción corporal, experiencial y cognitiva. Por tanto, no hablamos solamente de cognición situada, sino de acción situada en un contexto que se percibe como singular (Pérez Gómez, 20I2). Por otro lado, no podemos confundir esta acción con la importancia de la actividad por la actividad, sino que esta acción transforma la actividad como relevante para el propio sujeto y su proyecto vital. Como ya mencionábamos, el enactivismo encuentra su fundamento en una ontología relacional, ya que entiende los conocimientos como inter- y codependientes en relación de unos con otros (Gergen, 1998, 200I; Varela et al., 199I). Esto nos lleva a entender el conocimiento como el resultado de la interacción dinámica entre el agente y su contexto, por lo que se deduce, por un lado, que el conocimiento no es un proceso interno ni una representación fiel de la realidad; por otro, que la unidad de análisis debe ser la interacción permanente mente-contexto (Pérez Gómez, 2012).

Estas dinámicas de aula descritas son completamente coherentes con la idea de trabajo democrático que pretende el Movimiento para desarrollar una práctica de inclusión educativa en la que se incluya a todo el alumnado sea cual sea su necesidad educativa específica o dificultad de aprendizaje (López Melero, 201I). El éxito que tiene esta práctica evidencia que es posible construir una escuela sin exclusiones, inclusiva, mediante el respeto por las diferencias individuales y bajo la convicción de que todos son capaces de progresar y cumplir los objetivos establecidos y se contrapone a la idea de integración, bajo la que subyace una idea de cultura hegemónica a la que hay que adaptar la diferencia entre personas y a los colectivos minoritarios (López Melero, 2006).

Al final de la mañana los niños y las niñas se sientan en rueda de nuevo y señalan las tareas completadas durante el día a modo de evaluación. En la etapa preescolar no está establecido que exista calificación, sino que se evalúa día a día el trabajo en clase. Esta forma de evaluación entiende este proceso como un fenómeno moral en el que importa saber a qué valores sirve y a qué personas beneficia (Santos Guerra, 20I4), de manera que, al contrario que la calificación, la evaluación se ponga a favor y al servicio del aprendizaje por parte de todos los agentes que intervienen en el proceso evaluador (Alcaraz, 20I5), ya que la evaluación no es más que otra ocasión de aprendizaje en el aula (Álvarez Méndez, 2008), por lo que ha de ir más allá de una concepción tecnocrática de la enseñanza y repensar el significado de evaluar como sinónimos de investigar, conocer, analizar, comprender, mejorar, educar, formar... Esta evaluación se pone tanto al servicio del que aprende como de quien enseña, ya que recoge (y devuelve) información sobre los procesos y contextos del aula que ayuda a conocer, analizar y comprender en mayor profundidad el proceso de enseñanza-aprendizaje, que puede servir para mejorar la calidad del aprendizaje de todos los agentes (Alcaraz, 20I5). Una evaluación entendida así, entonces, se enfoca en el cómo se aprende, por lo que no se fija solo en el rendimiento del alumnado, sino también en la calidad de los contextos de aprendizaje que, como hemos visto, están especialmente cuidados en el Movimiento, ya que entendemos que una de las competencias básicas del docente es la preparación de las condiciones del aula y la observación permanente de las interacciones y relaciones entre el alumnado. Esto ayudará al docente en la planificación de su actuación y reestructurarla de acuerdo con las situaciones producidas en el contexto educativo (De la Blanca y Hidalgo, 20I2). 
En este momento del final del día se ofrece también un espacio para compartir las diferentes tareas que se hayan enviado para realizar con las familias. Los entornos familiares, además de otras actividades de aprendizaje social menos regladas, se muestran más flexibles y próximos a las nuevas formas culturales de aprendizaje. Por lo que no debe extrañarnos que se tomen de referencia estos escenarios de aprendizaje informal en el aula (Pozo, 2016). Estos contextos de aprendizaje son más complejos, inciertos y multidimensionales, por lo que permiten aprender indagando (Pérez Gómez, 20I2). Además, el papel de las familias es fundamental, aunque en la actualidad varios factores hayan difuminado las fronteras del papel de la escuela y las familias en la educación, como la evolución de las estructuras sociales, la extensión de la escolarización más temprana y la mayor capacitación de padres y madres. Sin embargo, los padres y las madres tienen el privilegio de entender mejor que nadie el comportamiento de su hijo/a, de saber interpretar sus reacciones ante cualquier circunstancia o estímulo (Corchete, 20I4). Es por ello por lo que desde la escuela se invita a la participación de las familias en el aula para que compartan las experiencias de aprendizaje con sus hijos/as.

Las normas de convivencia se deciden entre todos a través de la problemática que va surgiendo también durante este tiempo: en un cartel el alumnado, o un adulto que ayuda, escribe lo que le ha gustado y lo que no le ha gustado del comportamiento de sus compañeros y compañeras. Es decir, se recogen datos para que todos puedan participar en la regulación de las relaciones humanas en el aula. Al final de la semana, en una reunión organizada con ese fin, se pueden establecer normas, previa negociación con el grupo, a partir de estas afirmaciones. Normas que contribuirán a la regulación de las relaciones sociales en el grupo clase, entendido como microsociedad que decide sobre el modo de su relación. En esa reunión semanal también se analiza, con vistas a su regulación, la evolución de los aprendizajes individualmente y de forma colectiva. Con esta práctica entendemos que la ética se constituye cuando nos preocupamos por las consecuencias de nuestras acciones sobre otro y adquiere su forma al legitimar al otro en la convivencia (Maturana, 1994).

\section{Conclusiones}

Las dimensiones fundamentales y más ricas que conseguimos detectar en esta estrategia pedagógica que soporta esta organización social para aprender están relacionadas con conceptos y valores de cooperación, desarrollo de la autonomía y participación.

Un concepto «de moda» actualmente es la pedagogía diferenciada, la estrategia más adecuada para lidiar con la heterogeneidad, carácter intrínseco de los grupos de alumnos, siempre existente pero hoy más conscientemente presente. La apuesta de la pedagogía del Movimiento de la Escuela Moderna no niega su importancia. No obstante, continúa subrayando el papel destacado que atribuye al principio de cooperación que atraviesa la propuesta de la pedagogía diferenciada.

En este sentido se propone una escuela en que se viva en democracia, convivencia y respeto. Sin convivencia ni participación no puede darse el crecimiento humano y el aprendizaje (López Melero, 2006). Por eso es fundamental la construcción progresiva y participada de una organización social, mejor dicho, cooperativa, de los 
aprendizajes. Su condición de cooperativa o social le será reconocida por los diversos momentos y modalidades de implicación del alumnado en decisiones importantes de su itinerario de aprendizaje y en la organización para aprender. La democracia supone un espacio de convivencia en el respeto mutuo y la colaboración o, lo que es lo mismo, la preocupación por otros. Solo en este espacio puede surgir el ethos democrático, es decir, el reconocimiento de un individuo moral, cultural y socialmente autónomo (Cortina, 1997).

Una de las características más salientes del mundo contemporáneo es la gran producción y rapidez de circulación del conocimiento. Es decir, de los resultados de investigación científica. Muchas de las nuevas tareas académicas requieren nuevos conocimientos, habilidades y destrezas. Además, debemos poner en práctica estos aspectos en espacios sociales diversos, con nuevas conductas, actitudes y valores. No solo se trata de afrontar estas nuevas demandas, sino que tenemos que aprender a convivir con las múltiples identidades que creamos al desplegar tantas ideas, conocimientos, habilidades, actitudes, sentimientos o formas de vivir en los diversos contextos (Pozo, 20I6), que son todas las dimensiones del ser humano. Así, entenderíamos que todo aprendizaje relevante supone un acto de reinvención. En primer lugar, al apropiarnos de un contenido ya conocido por otros, en cierta manera siempre lo redefinimos, lo matizamos y lo singularizamos. En segundo lugar, porque aprender significa incorporar nuevos significados que fuerzan a reestructurar los anteriores, a reinventarse a uno mismo. En palabras de Pozo (2008) «aprender es siempre jugarse la identidad». Por eso, fueron gradualmente perdiendo sentido y utilidad las estrategias de transmisión de contenidos, como herramienta fundamental de la escuela, en cualquier nivel de enseñanza debido, entre otras características, a la inmensa acumulación de conocimientos. Las propuestas pedagógicas más avanzadas sugieren la apuesta por el desarrollo de la autonomía en la detección de fuentes de conocimiento; la selección de información, su tratamiento y organización, y la comunicación de lo aprendido, de manera que utilicemos los contenidos no como fin en sí mismos, sino como medio para desarrollar o construir nuevas capacidades (Pozo, 2016). Esto es la competencia de aprender a aprender.

El dominio de estrategias de aprender en ámbitos de autonomía es imprescindible en las pedagogías contemporáneas, sobre todo porque aprender es una de las actividades sociales a la que más tiempo dedicamos y que, a su vez, define nuestra identidad personal y social (Pozo, 20I6). Además, el aprendizaje relevante, explícito y comprensivo requiere la participación voluntaria del aprendiz y tiene dos pilares fundamentales como base: autonomía y responsabilidad (Pérez et al., 2009). Las nuevas escuelas tienen el deber de formar a los ciudadanos y ciudadanas en la utilización eficaz y creativa de la información que rodea y llena sus vidas. En el mundo contemporáneo, como apuntábamos, no es tan importante el volumen de conocimientos, sino la capacidad de tratarlos y aplicarlos en diferentes situaciones y contextos, en virtud de valores e intenciones de los propios proyectos personales o sociales (Pérez Gómez, 20Iz). Aprendemos de forma relevante cuando adquirimos significados que consideramos útiles para nuestros propósitos vitales. Este concepto de utilidad se relaciona estrechamente con el concepto de sentido, es decir, consideraremos útil aquello que ofrece un sentido para clarificar y afrontar los problemas básicos de nuestra vida y, por tanto, amplía nuestros horizontes de conocimientos, sensibilidades y afectos. Podríamos afirmar que el aprendizaje relevante sucede en una compleja red de intercambios que 
implica todas las dimensiones de nuestra personalidad (Pérez, Soto, Sola y Serván, 2009), ya que en el complejo proceso de la construcción del conocimiento intervienen, inevitablemente, componentes cognitivos, motivacionales, afectivos y sociales.

La tercera dimensión con carácter de fundamental en esta propuesta pedagógica es la participación. La participación del alumnado en diversas dimensiones de la vida escolar y educativa. Y también en la vida social. Al fin y al cabo, la adquisición eficaz de habilidades, conductas, actitudes y conocimientos forma parte de un proceso de familiaridad con formas de ser, pensar, sentir y percibir característicos del grupo y el entorno en el que se desenvuelve nuestra vida (Lave y Wenger, 199I). Por eso la apuesta del MEM en la construcción de una democracia directa comenzando por la escuela. Para que tenga continuidad en la vida social más alargada. El alumnado de las salas que fueron observadas participa en la planificación de lo que van a aprender, porque, como afirma Pozo (2008), «conjugar juntos los verbos enseñar y aprender es sintonizar la actividad cognitiva de aprendices y maestros». Se implican en la ejecución de lo planeado y constituyen una pieza decisiva, por su participación, en el proceso de evaluación. En portugués existe un dicho muy ilustrativo de lo que algunos piensan cuando contactan con este tipo de estrategias participativas que convocan la implicación de los alumnos: «dar el oro a los bandidos» (Dar o ouro ao bandido).

En todas las democracias sanas y honestas no existen espacios para el abuso y exageración por parte de algunos. Cuando surgen o acontecen, el propio sistema democrático tiene mecanismos para subsanarlo. Lo mismo acontece en esta experiencia de democracia directa en el aula. Además de participar en el proceso de aprendizaje (organización social de los aprendizajes), también ellos participan y toman decisiones en aspectos que tengan que ver con la gestión de las relaciones humanas en ese grupo de personas. Los conflictos de relación entre ellos y con el docente (indisciplina, para algunos) son analizados por todos para tomar decisiones. De allí surgen las reglas de convivencia del grupo de esa aula.

Con este artículo se quiere poner en manifiesto el rol que juega la escuela cuando toma consciencia del contexto en el que vive y se siente en el deber de actuar en consecuencia con su alumnado. Así, la sociedad construye la escuela, aunque también actúa en consecuencia en la dirección opuesta: la escuela participando y, por tanto, construyendo en y para la sociedad.

\section{Bibliografía}

Alcaraz, N. (2015). Evaluación versus calificación. Aula de Encuentro, 2(17), 209-236.

Álvarez Méndez, J. M. (2008). Evaluar el aprendizaje en una enseñanza centrada en las competencias. En J. Gimeno, Educar por competencias ¿qué hay de nuevo? Madrid: Morata.

Clemente, M. y Rodríguez, I. (20I4). Enseñanza inicial de la lengua escrita. De la teoría a la práctica. Aula. Revista de Pedagogía de la Universidad de Salamanca, 20, 25-44.

Corchete, T. (20I4). La familia, un aliado indispensable para fomentar la lectura desde los espacios profesionales. Aula. Revista de Pedagogía de la Universidad de Salamanca, 20, I23-I32.

Cortina, A. (1997). Ciudadanos del mundo. Madrid: Alianza.

De La Blanca, S. e Hidalgo, J. (20I2). Formación inicial. Nuevos maestros para tiempos nuevos. Revista Padres y Maestros, (345), 20-24. 
Defior, S. (20I4). Procesos implicados en el reconocimiento de las palabras escritas. Aula. Revista de Pedagogía de la Universidad de Salamanca, 20, 25-44.

Gergen, K. (1998). La psicología social como historia. Revista Antropos, 177, 39-49.

GERGEN, K. (200I). Social constructions in context. Londres: Sage.

GibBs, G. (2012). El análisis de datos cualitativos en investigación cualitativa. Morata.

Goezt, J. y Lecompte, M. (1988). Etnografía y diseño cualitativo en investigación. Madrid: Morata.

González Roa, P. (2000). El movimiento de la escuela moderna portuguesa. Historia de la educación. Revista Interuniversitaria, (I9), 3II-329.

Kegan, R. y LASKow, L. (20IO). Immunity to change. How to unlock the potential in yourself and your organization. Cambridge, MA: Harvard Business Review Press.

Lave, E. y Wenger, E. (I99I). Situated Learning. Cambridge, Mas: Cambridge University Press.

López Melero, M. (2006). La ética y la cultura de la diversidad en la escuela inclusiva. Tavira, $(2 I), 9-25$.

López Melero, M. (20II). Barreras que impiden la escuela inclusiva y algunas estrategias para construir una escuela sin exclusiones. Innovación Educativa, 2I, 37-54.

Maturana, H. (1994). El sentido de lo humano. Santiago de Chile: Dolmen.

NizA, S. (1992). Em comum Assumimos uma Educação Democrática. En M. Henrique, G. Vilhena y J. Soares, Cadernos de Formação Cooperada, n. ${ }^{\circ}$ I (pp. 39-47). Lisboa: Movimento da Escola Moderna.

Niza, S. (1996). O Modelo Curricular da Educação Pré-Escolar da Escola Moderna Portuguesa. En Modelos curriculares para a Educação de Infância (pp. 139-159). Porto: Porto Editora.

Pérez, Á. I.; Soto, E.; Sola, M. y Serván, M. J. (2009). Aprender cómo aprender. Autonomía y responsabilidad: el aprendizaje de los estudiantes. Madrid: Ediciones Akal.

Pérez Gómez, A. I. (2012). Educarse en la era digital. Madrid: Morata.

Pires, J. (1995). Práticas de Planificação na Escola Moderna Portuguesa. Universidad de Lisboa.

Pozo, I. (2008). Aprendices y maestros. Madrid: Alianza Editorial.

Pozo, I. (2016). Aprender en tiempos revueltos. Madrid: Alianza Editorial.

Premarck, D. y Premarck, A. J. (1996). Why animals lack pedagogy and some cultures have more of it than others. En D. Olson y N. Torrance (eds.) Handbook of Educaction and Human Development. Oxford: Blackwell.

Pressley, M.; Wharton-McDonald, R.; Allington, R.; Collins-Block, C.; Morrow, L.; Tracey, D. y Woo, D. (200I). A study of effective first-grade literacy instruction. Scientific studies of reading, $5(\mathrm{I}), 25-58$.

Santos Guerra, M. A. (20I4). La evaluación como aprendizaje. Cuando la flecha impacta en la diana. Madrid: Narcea.

Simons, H. (20II). El estudio de caso: teoría y práctica. Madrid: Morata.

Tomasello, M. (2009). Why we cooperate. Cambridge: The mit Press.

Varela, F. J.; Thompson, E. y Rosch, E. (199i). The embodied mind: cognitive science and human experience. Cambridge, MA: MIT Press. 
